underestimation might be a problem and therefore needs further examination in famine settings. In studies of the Ukraine Holodomor famine of 1932-1933 and of the Dutch Hunger Winter famine of 1944-1945, we demonstrated that there was no difference in type 2 diabetes mellitus (T2DM) when we compared individuals born before the famine (and hence exposed during childhood) with individuals born after the famine ${ }^{3,4}$. We therefore combined these groups to form an age-balanced control group. We further addressed the age issue with difference in difference analytic methods, comparing the effect of famine in different severity areas. Findings in our Dutch famine study were also confirmed using same-sex siblings without famine exposure as controls ${ }^{1}$. These approaches protect from possible biases arising from the rapidly increasing prevalence of T2DM in these settings and in the Chinese population in the past few years.

Our problem with a recent study ${ }^{5}$ mentioned by Zimmet et al. in support of a possible interaction between famine exposure and the risk of hyperglycaemia and cardiovascular disease is that the study failed to use age-balanced controls. This omission probably inflated the estimated risk of hyperglycaemia following exposure to famine and thereby inflated estimates of any further interactions with hyperglycaemia.

Zimmet et al. question whether metaanalysis is an appropriate tool to help synthesize findings from a multitude of Chinese famine studies and T2DM risk. We used metaanalysis in the first place to identify differences in the type of controls used across studies. This approach shows that most studies failed to use age-balanced controls and reported falsepositive findings. Subsequently, we described consistent patterns across all studies, regardless of famine exposure definition or of T2DM assessment methods. When age-balanced controls are used, the studies to date fail to show an association between prenatal famine exposure and later life T2DM. Adjustment for covariates - as is conducted in some studies - does not change this pattern. If the Chinese Famine had a long-term impact on T2DM, the failure to demonstrate this in most Chinese Famine studies is most likely due to classification errors in the timing or severity of famine exposure and perhaps also to the limited range of exposure in the studied populations.

In their response, Zimmet et al. state they excluded studies published in Chinese because "these studies were conducted in regions severely affected by famine $[. .$.$] and$ did not include less severely affected regions as controls". We see no justification for this omission, as studies published in Chinese accounted for half of all published famine studies and covered several regions with appropriate famine controls ${ }^{2}$. Indeed, most of these studies had similar designs to those published in English. Ignoring these Chinese studies will give an incomplete, and possibly biased, picture of the findings on this topic. Zimmet et al. also seem to uncritically accept what is claimed about the severity of famine in selected study populations. As an example, the Suihua Beilin area of Heilongjiang province was classified as 'a severe famine area' by the study's authors ${ }^{6}$; however, based on demographic and population census data at either the prefecture or the provincial level, the Heilongjiang province was one of the provinces least affected by the famine compared with others ${ }^{7}$. The famine severity classification of the study was entirely based on the reported loss of grain production in the area and not on any other data. This information was not further discussed by the study authors.

The final point made by Zimmet et al. is that, in a recent study published in 2019 (REF. ${ }^{8}$ ), early-life exposure to the Chinese Famine increased IGF2 gene methylation in adulthood, 'agreeing with findings from the Dutch famine' as reported by us ${ }^{9}$. We see no agreement with Dutch findings, however, as only one CpG in the cited study shows an association in the desired direction and all others show effects in the opposite direction.

Chihua Li ${ }^{1,2}$, Elmar W. Tobi $i^{3,4}$ Bastiaan T. Heijmans ${ }^{3}$ and L. H. Lumey (iD) ${ }^{1,3 *}$ Department of Epidemiology, Mailman School of Public Health, Columbia University, New York, NY, USA
2Zhengzhou Central Hospital Affiliated to Zhengzhou University, Henan, China.

${ }^{3}$ Molecular Epidemiology, Department of Biomedical Data Sciences, Leiden University Medical Center, Leiden, Netherlands.

${ }^{4}$ Division of Human Nutrition, Wageningen University and Research, Wageningen, Netherlands. *e-mail: lumey@columbia.edu https://doi.org/10.1038/s41574-019-0301-8

1. Zimmet, P. et al. Chinese famine and the diabetes mellitus epidemic. Nat. Rev. Endocrinol. https:/ doi.org/10.1038/s41574-019-0300-9 (2019).

2. $\mathrm{Li}, \mathrm{C}$. et al. The effect of the Chinese Famine on type 2 diabetes mellitus epidemics. Nat. Rev. Endocrinol. 15 313-314 (2019).

3. Lumey, L., Khalangot, M. D. \& Vaiserman, A. M. Association between type 2 diabetes and prenatal exposure to the Ukraine famine of 1932-33: a retrospective cohort study. Lancet Diabetes Endocrinol. 3, 787-794 (2015).

4. Lumey, L., Stein, A. \& Kahn, H. Food restriction during gestation and impaired fasting glucose or glucose tolerance and type 2 diabetes mellitus in adulthood: evidence from the Dutch Hunger Winter Families Study. J. Dev. Orig. Health Dis. 1, S164 (2009).

5. Zhang, Y. et al. Exposure to Chinese famine in early life modifies the association between hyperglycaemia and cardiovascular disease. Nutr. Metab. Cardiovasc. Dis. 29, 1230-1236 (2019)

6. Li, J. et al. Prenatal exposure to famine and the development of hyperglycemia and type 2 diabetes in adulthood across consecutive generations: a population-based cohort study of families in Suihua, China. Am. J. Clin. Nutr. 105, 221-227 (2016).

7. Li, C. $\&$ Lumey, L. Exposure to the Chinese famine of 1959-61 in early life and long-term health conditions a systematic review and meta-analysis. Int. J. Epidemiol. 46, 1157-1170 (2017)

8. Shen, L. et al. Early-life exposure to severe famine is associated with higher methylation level in the IGF2 gene and higher total cholesterol in late adulthood: the Genomic Research of the Chinese Famine (GRECF) study. Clin. Epigenetics 11, 88 (2019).

9. Heijmans, B. T. et al. Persistent epigenetic differences associated with prenatal exposure to famine in humans. Proc. Natl Acad. Sci. USA 105 17046-17049 (2008).

\title{
Early-life exposure to the Chinese Famine and subsequent T2DM
}

\section{Zhiyong Zou (D), Changwei Li and George C. Patton (1)}

Over the past 10 years, 17 studies have reported that antenatal exposure to the Chinese Famine across the first 3 years of life increased the subsequent risk of type 2 diabetes mellitus (T2DM) and/or hyperglycaemia. A 2018 review also concluded that the famine has contributed to China's current T2DM epidemic ${ }^{1}$. However, in a Comment published in Nature Reviews Endocrinology (Li, C. et al. The effect of the Chinese Famine on type 2 diabetes mellitus epidemics. Nat. Rev. Endocrinol. 18, 313-314 (2019) $)^{2}$, which included discussion of a 2017 meta-analysis of published Chinese famine studies using age-matched controls ${ }^{3}$, Chihua $\mathrm{Li}$ and colleagues found that famine was not associated with an increased risk of T2DM.
We agree with $\mathrm{Li}$ and colleagues that both the age of assessment and severity of exposure contribute to the differences in findings. In the 2002 China National Nutrition and Health Survey (CNNHS), the mean ages for participants in the non-exposed, fetal-exposed, early-childhood-exposed, mid-childhoodexposed and late-childhood-exposed cohorts were 39, 42, 45, 47 and 49 years, respectively ${ }^{4}$. Similarly, in the Survey on Prevalence in East China of Metabolic Diseases and Risk Factors (SPECT-China), the mean ages for the non-exposed, fetal-exposed, childhood-exposed and adolescence-exposed or adult-exposed cohorts were 40-51, $52-55,56-65$ and $66-93$ years, respectively ${ }^{5}$. 
Such differences in ages of assessment will undoubtedly have affected the likelihood of receiving a diagnosis.

Equally important, Chinese Famine exposure varied substantially, indicated by very different mortality across provinces ${ }^{6}$. Such differences in exposure will have effects on the association with $\mathrm{T}_{2} \mathrm{DM}^{4,7,8}$. In the severely affected famine areas in the CNNHS study, fetal-exposed participants had a clear increased risk of hyperglycaemia (OR 3.92; 95\% CI 1.64-9.39), but no risk was apparent in less severely affected areas

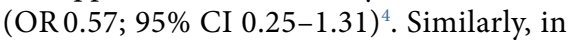
the SPECT study in Shandong and Anhui provinces, we again found that severe fetal exposure to famine resulted in an increased risk of T2DM (OR 1.59; 95\% CI 1.11-2.30) but found no association in less severely affected areas ${ }^{8}$.

Moreover, in our 2017 report from the China Health and Retirement Longitudinal Survey (CHARLS), fetal exposure to famine also resulted in an increased risk of T2DM after participants were age-matched (combination of the non-exposed cohort 1962-1964 and pre-famine cohort 1956-1958) (OR 1.37; 95\% CI $1.07-1.75)^{9}$. Given that CHARLS is a nationally representative sample of those $>45$ years of age, we were surprised that it was not included in the meta-analysis.

In this context, we re-analysed six studies (five that were also included in the Review by Zimmet et al. and one from the CHARLS) with age-matched controls ${ }^{1,8,9}$, taking into account severity of famine exposure. The summary estimate showed small but significant overall effects on the risk of T2DM (OR 1.25; 95\% CI 1.15-1.37). However, we found a clearer association in severely affected areas (OR 1.38; 95\% CI 1.11-1.72) and no association in less severely affected areas (OR 0.94; 95\% CI 0.57-1.57) (Supplementary Tables).

Based on these findings, we conclude that the severity of antenatal exposure to the Chinese Famine determined heightened risk for later T2DM. Given that severe exposure was widespread in China (15 of 28 provinces had an over $50.0 \%$ higher mortality than the 3 years (1956-1958) immediately preceding the famine (1959-1961) $)^{6,9}$ ), it seems likely that the Chinese Famine has indeed substantially contributed to the current T2DM epidemic. We agree with $\mathrm{Li}$ and his colleagues that, regardless of earlier famine exposure, healthy lifestyles will reduce the burden of T2DM, but this does not diminish the significance of the Chinese Famine effects.

There is a reply to this letter by $\mathrm{Li}, \mathrm{C}$. et al. Nat. Rev. Endocrinol. https://doi.org/10.1038/ s41574-019-0302-7 (2019).
Zhiyong Zou (iD ${ }^{1 *}$, Changwei $\mathrm{Li}^{2}$ and George C. Patton iD 1,3,4,5 ${ }^{1}$ Institute of Child and Adolescent Health, Peking University School of Public Health; National Health Commission Key laboratory of Reproductive Health, Peking University, Beijing, China. Department of Epidemiology and Biostatistics, College of Public Health, University of Georgia, Health Sciences Campus, Athens, GA, USA.

${ }^{3}$ Department of Paediatrics, Faculty of Medicine The University of Melbourne, Parkville, Victoria, Australia.

${ }^{4}$ Centre for Adolescent Health, Royal Children's Hospital, Parkville, Victoria, Australia. ${ }^{5}$ Murdoch Children's Research Institute, Parkville, Victoria, Australia. *e-mail: harveyzou2002@bjmu.edu.cn https://doi.org/10.1038/s41574-019-0299-y

1. Zimmet, P. et al. Epidemic T2DM, early development and epigenetics: implications of the Chinese Famine. Nat. Rev. Endocrinol. 14, 738-746 (2018).

2. Li, C. et al. The effect of the Chinese Famine on type 2 diabetes mellitus epidemics. Nat. Rev. Endocrinol. 18 313-314 (2019)

3. Li, C. $\&$ Lumey, L. H. Exposure to the Chinese famine of 1959-61 in early life and long-term health conditions: a systematic review and meta-analysis. Int. J. Epidemiol. 46, 1157-1170 (2017).

4. Li, Y. et al. Exposure to the Chinese famine in early life and the risk of hyperglycemia and type 2 diabetes in adulthood. Diabetes 59, 2400-2406 (2010).

5. Wang, N. et al. Is exposure to famine in childhood and economic development in adulthood associated with diabetes? J. Clin. Endocrinol. Metab. 100, 4514-4523 (2015).

6. Luo, Z., Mu, R. \& Zhang, X. Famine and overweight in China. Rev. Agr. Econ. 28, 296-304 (2006).

7. Li, J. et al. Prenatal exposure to famine and the development of hyperglycemia and type 2 diabetes in adulthood across consecutive generations: a population-based cohort study of families in Suihua, China. Am. J. Clin. Nutr. 105, 221-227 (2017).

8. Wang, N. et al. Exposure to severe famine in the prenatal or postnatal period and the development of diabetes in adulthood: an observational study. Diabetologia 60, 262-269 (2017).

9. Wang, Z. et al. The association between fetal-stage exposure to the China famine and risk of diabetes mellitus in adulthood: results from the China health and retirement longitudinal study. BMC Public Health 18, 1205 (2018)

\section{Acknowledgements}

The authors acknowledge the support of the National Natural Science Foundation of China $(81402692$; 81773454), the China Scholarship Council (201806015008) and the Research Special Fund for Public Welfare Industry of Health of the Ministry of Health of China (201202010). We acknowledge J. Ma, Y. Ma and Z. Wang for their help preparing this article and discussion of the content.

\section{Competing interests}

The authors declare no competing interests.

Supplementary information

Supplementary information is available for this paper at https://doi.org/10.1038/s41574-019-0299-y.

\section{Reply to 'Early-life exposure to the Chinese Famine and subsequent T2DM'}

\section{Chihua Li, Elmar W. Tobi, Bastiaan T. Heijmans and L. H. Lumey}

We thank Zou et al. (Early-life exposure to the Chinese Famine and subsequent T2DM. Nat. Rev. Endocrinol. https://doi.org/10.1038/ s41574-019-0299-y (2019) ${ }^{1}$ for their interest in our Comment $(\mathrm{Li}, \mathrm{C}$. et al. The effect of the Chinese Famine on type 2 diabetes mellitus epidemics. Nat. Rev. Endocrinol. 15, 313-314 $(2019))^{2}$ and welcome the opportunity to address their concerns about the completeness of the data we presented.

Zou et al. re-analysed the five studies included in a Review by Zimmet et al. ${ }^{3}$ together with their own study that combined prefamine and post-famine controls from the 2011 China Health and Retirement Longitudinal Study ${ }^{4}$. They claim a statistically significant overall effect on the risk of type 2 diabetes mellitus (T2DM) from an odds ratio of 1.25 (95\% CI 1.15-1.37) for all studies combined using age-balanced controls and a randomeffects model. Using the data as provided by Zou et al. in their supplementary table 1 (REF. ${ }^{1}$ ), we undertook the same analysis, including their added study, and still find a non- significant overall effect on the risk of T2DM with an odds ratio of 1.05 (95\% CI 0.87-1.26). A summary of our calculations is attached (Supplementary Fig. 1).

For their re-analysis of studies in severely affected famine areas, Zou et al., in our view, should have been more careful in their choice of studies. The findings from the included Suihua cohort show an increase in T2DM in famine births compared with post-famine births ${ }^{5}$, but demographic and population census data suggest that the study area was in one of the provinces least affected by the famine ${ }^{6,7}$. This calls for a re-examination of their findings on severe famine exposure effects.

We do not dispute that Zou et al. could be correct in believing that the famine had a marked effect on current and future T2DM epidemics in China. This conclusion, however, cannot be drawn from current studies. For example, the Ukraine and Dutch famines had a statistically significant effect on later $\mathrm{T}_{2} \mathrm{DM}^{8,9}$, and the same could be true in China. Ideally this would be demonstrated 